\title{
Gramática lúdica y creativa. Una experiencia para hacer más accesibles los contenidos gramaticales en Educación Primaria ${ }^{1}$
}

Ludic and creative grammar. An experience to make grammatical contents in Primary Education more accessible

\author{
Sergio Suárez Ramírez ${ }^{2}$ \\ Universidad de Valladolid, España \\ sergiosuarezramirez@gmail.com \\ https://orcid.org/0000-0002-8050-2590 \\ Marta Mateos Núñez \\ Universidad de Valladolid, España \\ martamateosnunez@gmail.com \\ https://orcid.org/0000-0001-7784-9019 \\ Miriam Suárez Ramírez \\ Universidad de Extremadura, España \\ myrysuarez@hotmail.com \\ https://orcid.org/0000-0001-8438-8505
}

\section{Resumen:}

La enseñanza de la lengua y la literatura ha de ser una experiencia lúdica y sensorial para el alumnado, sobre todo a la hora de desarrollar contenidos curriculares referidos a la reflexión sobre la propia lengua y la gramática. Este trabajo expone una investigación empírica llevada a cabo

\begin{abstract}
:
Teaching language and literature should be a ludic and sensorial experience for students, especially when it comes to developing curricular content targeting students' own reflection about their language and grammar. Data were obtained through the completion of creative and playful activi-
\end{abstract}

1 Como referenciar este artículo (How to reference this article):

Suárez Ramírez, S., Mateos Núñez, M., \& Suárez Ramírez, M. (2021). Gramática lúdica y creativa. Una experiencia para hacer más accesibles los contenidos gramaticales en Educación Primaria. Educatio Siglo XXI, 39(3), 187-208. https://doi.org/10.6018/ educatio. 427811

2 Dirección para correspondencia (Correspondence address):

Sergio Suárez Ramírez. Departamento de Didáctica de la Lengua y la Literatura. Facultad de Educación. Avd. Duques de Soria s/n. 42004, Soria (España). 
Suárez Ramírez, S., Mateos Núñez, M. y Suárez Ramírez, M. (2021). Gramática lúdica y creativa. Una experiencia para hacer más accesibles los contenidos gramaticales en Educación Primaria.

Educatio Siglo XXI, 39(3), 187-208.

mediante el trabajo de campo y la realización de actividades lúdicas en torno a los usos del lenguaje, desarrollada primero con 18 alumnos del Grado de Maestro de la Facultad de Educación de Soria (Universidad de Valladolid) y, después, con 274 escolares de seis centros de Educación Primaria de la ciudad donde desarrollan sus prácticas curriculares. La experiencia consistió en la realización, bajo la supervisión de sus tutores, de actividades a modo de juegos creativos en tres niveles lingüísticos: letras, palabras y textos. Los objetivos planteados fueron: despertar la curiosidad y el sentido critico del alumnado participante en la experiencia, tanto universitario como de la etapa de Educación Primaria; propiciar la reflexión sobre el código lingüístico para mejorar la expresión oral y escrita de los escolares; y facilitar la asimilación de diversos contenidos curriculares de forma libre, autónoma y cooperativa. Al presentarse como juegos, pudieron aplicarse con igual rendimiento en estas dos etapas educativas (Primaria y Universidad). En su realización, los errores se contemplaron como una oportunidad creativa. Los resultados obtenidos confirman el valor de la enseñanza de la gramática de forma lúdica y creativa. La mayoría de los escolares de Educación Primaria mejoró los resultados en cuanto a esfuerzo, corrección lingüística o lectura en voz alta; por su parte el alumnado universitario apreció la importancia de incluir recursos lúdicos en los procesos de enseñanza y aprendizaje, al repercutir sobre una mejor actitud afectiva del alumnado, reforzando la importancia de la sugestopedia como metodología en la enseñanza lingüística.

\section{Palabras clave:}

Creatividad; gramática; enseñanza; lúdica; lenguaje. ties about language, developed first with 18 students of Education Degree in Soria (University of Valladolid) and, later, with 274 students from six Primary schools where university students developed their curricular practices. The experience consisted of the design and conduction, under school tutors' supervision, of creative games which targeted three linguistic levels: letters, words and texts. More specifically, the objectives included: to awake learners' curiosity and critical thinking; to promote reflection on the linguistic code for improving oral and written expression; and to facilitate the assimilation of several grammatical contents freely, autonomously and cooperatively. Since these activities were presented as games, they were applied with the same performance levels at university and at primary school settings. Mistakes were considered opportunities for creativity. The results obtained confirm the value of teaching grammar in a playful and creative way. Most of the Primary students improved their results in terms of effort, linguistic correction and reading aloud. University students highlighted the importance of including recreational resources in teaching and learning processes as this had a positive effect on primary school learners' affective attitude. The results obtained reinforce the importance of suggestion-pedagogy as a method in language teaching.

\section{Key words:}

Creativity; grammar; teaching; playful; language.

\section{Résumé:}

L'enseignement des langues et de la littérature doit être une expérience ludique et sensorielle pour les élèves, notamment lorsqu'il s'agit de développer des contenus curriculaires liés à la réflexion sur la langue et la grammaire. Cet article présente une recherche empirique menée à travers un travail de terrain et la mise en place d'activités ludiques autour des usages de la langue, développées d'abord avec 18 étudiants de la Licence en Enseignement de la Faculté d'Éducation de Soria (Université de Valladolid) et, ensuite, 
avec 274 étudiants de six centres d'Éducation Primaire de la ville où ils développent leurs pratiques curriculaires. L'expérience consistait à réaliser, sous la supervision de leurs tuteurs, des activités sous forme de jeux créatifs à trois niveaux linguistiques : lettres, mots et textes. Les objectifs étaient les suivants : éveiller la curiosité et le sens critique des étudiants participant à l'expérience, qu'il s'agisse d'étudiants de l'université ou de l'école primaire ; encourager la réflexion sur le code linguistique afin d'améliorer l'expression orale et écrite des étudiants; et faciliter l'assimilation de différents contenus curriculaires de manière libre, autonome et coopérative. Comme ils étaient présentés comme des jeux, ils pouvaient être appliqués avec la même performance dans ces deux étapes éducatives (primaire et universitaire). Dans leur mise en œuvre, les erreurs étaient considérées comme une opportunité créative. Les résultats obtenus confirment lsintérêt dıenseigner la grammaire de manière ludique et créative. La majorité des élèves de lıécole primaire ont amélioré leurs résultats en termes dieffort, de correction linguistique ou de lecture à haute voix ; tandis que les étudiants universitaires ont apprécié lsimportance dsinclure des ressources ludiques dans les processus dıenseignement et diapprentissage, car elles ont un impact sur une meilleure attitude affective des étudiants, renforçant ainsi l simportance de la suggestopédie comme méthodologie dans lienseignement des langues.

\section{Mots-clés:}

Créativité; grammaire; enseignement; ludique; langue.

Fecha de recepción: 18-05-2020

Fecha de aceptación: 27-09-2020

\section{Introducción}

Todos sabemos que la infancia es una etapa de la vida en la que más se desarrolla el sentido creativo en los niños. La capacidad de recepción, la frescura de ideas, el poder de admiración, el instinto del juego, el gusto por el riesgo, los frecuentes e interminables porqués y las relaciones imprevistas e insólitas son características específicas de esta etapa educativa.

El juego contribuye a asimilar lo real sin coacciones ni sanciones (Piaget, 2007), a mejorar el desarrollo cognitivo (Vigotsky, a través de Nevado Fuentes, 2008) y a favorecer la imaginación (Dewey, a través de Nussbaum, 2012).

Hidalgo (2007) asocia la enseñanza y la creatividad con el pensamiento inventivo y sugiere el uso de estrategias que orienten rigurosamente al docente y al estudiante en el trabajo. También propone un enfoque que integre diseño, secuencia de contenidos y procesos apropiados para desarrollar conductas y actitudes basadas en los principios de la creatividad.

Para Fueguel y Montolíu (2005) la creatividad no debe reducirse sólo 
a las materias artísticas, ni ser patrimonio de unos pocos privilegiados, sino que tiene que ser un proceso habitual y abierto a todos. El uso de herramientas de pensamiento permite sacar al individuo de los extremos polarizados para explorar nuevos caminos.

Una propiedad de la personalidad creativa es el sentido lúdico. Muchas personas han puesto de relieve su actitud lúdica frente a los problemas de los que se ocuparon. Jugaban con el significado de hechos conocidos y por ahí encontraron soluciones inesperadas. Jugaban con los objetos, y así descubrieron propiedades y dimensiones desconocidas. Jugaban con elementos totalmente inconexos y hallaron nuevas relaciones entre ellos. Por eso, es importante resaltar el sentido lúdico de la vida, pues jugar físicamente con los objetos y jugar mentalmente con las ideas lleva al alumnado a ir adquiriendo una actitud creativa en educación.

Cada día es mayor la conciencia sobre el papel del juego en el proceso de enseñanza y aprendizaje de los niños. El juego es una actividad irremplazable para desarrollar la capacidad de aprendizaje de los niños, un medio de expresión tanto en el plano físico, como en el cognitivo, psicológico y social. Labrador y Morote (2008) consideran que:

\begin{abstract}
"Los juegos son tan viejos como el hombre, jugar ha sido una técnica de aprendizaje habitual a través de los tiempos, pero el reconocimiento de su valor educativo todavía tiene mucho camino por recorrer. Si observamos la historia de la educación, comprobaremos que ha servido para fomentar el trabajo en equipo, favorecer la sociabilidad del estudiante, y desarrollar la capacidad creativa, crítica y comunicativa del individuo" (p. 71).
\end{abstract}

A pesar de su corto recorrido, están ya muy desarrolladas las teorías que han puesto de relieve la importancia de la inteligencia emocional y cómo influye en el aprendizaje la actitud con que se afronte los diferentes contenidos (Bizquerra Alzina y Pérez Escoda, 2007; Pérez Escoda, Filella y Sildevila, 2005 o Rodríguez Moreno, 2006). Tampoco son nuevos los planteamientos y estudios que han destacado el valor de los juegos, en especial los que implican un uso creativo del lenguaje (García y Llull, 2009; Vilera, 2004; Martí, 2010; Lantigua, 2007; Bernal y Brito, 2006). Y los más recientes estudios que valoran la necesidad de la gamificación en los procesos de enseñanza-aprendizaje (Figueroa, 2015) para fomentar la comunicación y colaboración entre alumnos, con experiencias desarrolladas a partir de escape rooms (Gómez López, 2019), realidad 
virtual (Moreno, Leiva, Galván, López y García, 2017) o realidad aumentada (Sánchez Bolado, 2017).

\section{Lenguaje, creatividad y educación}

La creatividad aplicada a la enseñanza de la lengua debe hacer referencia a actividades, estrategias, recursos y metodologías que persigan el desarrollo de la competencia en comunicación lingüística de una forma lúdica, e incluya la competencia digital, y aquellas propias de la cultura emprendedora. Supone abrir en las aulas espacios de expresión para encontrar respuestas diversas y originales fomentando la imaginación y la creatividad del alumnado.

Labrador y Morote (2008) también han destacado la importancia de los juegos de lenguaje, al afirmar que todo hablante posee una actividad metalingüística inconsciente. Esta actividad se revela del todo en el juego: juegos de palabras, juegos con las palabras, juegos verbales bajo todas sus formas (retruécanos, jeroglíficos, charadas, lapsus burlescos de contraposición de letras, canciones infantiles para señalar a quién le toca hacer algo, adivinanzas, palabras compuestas, etc.); en resumen, todas esas manifestaciones de la palabra que testimonian entre los hablantes una lingüística innata, intuitiva, pues el jugar supone que se conocen las reglas y el medio de interpretarlas aprovechando la ambigüedad que caracteriza las lenguas naturales, así como la creatividad que permiten.

Las instituciones educativas europeas, nacionales y regionales, a través de sus directrices sobre educación, reclaman que los alumnos desarrollen la creatividad en las aulas con actividades que sirvan para entender el código lingüístico y que permitan que se comuniquen experimentando, a través de un aprendizaje significativo (aprender a aprender).

El Marco Común Europeo de Referencia de las Lenguas (MCER, 2002), en el capítulo 4 sobre "El Uso de la lengua y el usuario o alumno", en sus "Tareas y propósitos comunicativos" se refiere al componente lúdico que debe acompañar todo acto educativo. No debemos olvidar que mediante el juego el estudiante pasa a un papel activo, se convierte en el protagonista del proceso de aprendizaje. En la enseñanza es importante diseñar actividades donde el alumnado ponga en práctica los cincos sentidos y que experimente. Los profesores de lenguas tienen la oportu- 
nidad de presentar las actividades como juegos del lenguaje, de palabras, letras y textos, vertebrando el código lingüístico y haciendo hincapié en cómo pueden combinarse. De esta forma la aproximación posterior a los textos literarios será más amena y experimental, más manipulable, más lúdica.

La Ley Orgánica de Educación (LOE) de 2006 en su art. 16 del capítulo II sobre Educación Primaria dice que: "la finalidad de la educación primaria es proporcionar a todos los niños y niñas una educación que permita afianzar su desarrollo personal y su propio bienestar, adquirir las habilidades culturales básicas relativas a la expresión y comprensión oral, a la lectura, a la escritura y al cálculo, así como desarrollar las habilidades sociales, los hábitos de trabajo y estudio, el sentido artístico, la creatividad y la afectividad". Además, uno de los objetivos más importantes en esta etapa educativa es que los alumnos desarrollen "actitudes de confianza en sí mismos, su sentido crítico, iniciativa personal, curiosidad, interés y creatividad en el aprendizaje".

Consideramos que existe una estrecha relación entre el concepto de creatividad y el de competencia clave. Si se entiende por competencia la forma en la que cualquier persona a que utilice sus recursos personales (habilidades, actitudes, conocimientos y experiencias) para actuar de manera activa y responsable en la construcción de sus proyectos de vida, tanto personal como social. Es evidente que eso coincide con el concepto de creatividad, porque se refiere a la capacidad de las personas para proceder de manera diferente, para resolver problemas o abordar situaciones diversas.

Es importante seguir los consejos de Pisonero del Amo (2004) que insiste en la idea de tomar el juego como recurso en la enseñanza de lenguas. "Porque los juegos son, en especial en la infancia, elementos esenciales del proceso de aprendizaje" (p. 1295). Como docentes es necesario que la enseñanza sea algo ameno y entretenido en algunas ocasiones (aunque tampoco es bueno que siempre sea así). Dice Nevado Fuentes (2008, p. 6) que cuando "los discentes tienen la sensación de que aprenden sin esfuerzo", las actividades deben ser guiadas para convertirlas en motivantes y lúdicas.

Es evidente que la gramática crea un metalenguaje compartido entre el alumno y el profesor para dialogar sobre el objeto de aprendizaje (el código lingüístico). Su enseñanza posibilita el dominio de la norma lingüística, construida en buena parte sobre conceptos gramaticales. Al 
centrar la enseñanza de una lengua en la gramática se está favoreciendo que los alumnos puedan revisar y corregir sus propios usos, a la vez que se propicia una mejoren significativa en su cultura lingüística. No obstante, ninguna gramática es adecuada si se convierte en sí misma en el centro de aprendizaje. Es conveniente, por tanto, enseñar menos gramática de la que se enseña actualmente $\mathrm{o}$, al menos, enseñarla de una forma más lúdica y divertida, más práctica. La clave en las primeras etapas educativas es preguntarse ¿cuál es el objetivo que debe perseguir una gramática pedagógica? ¿qué deben saber los alumnos sobre su propia lengua para poderla utilizar con éxito fuera del aula?

Para lograr que la enseñanza de la gramática sea efectiva, los docentes tienen que asumir que no se trata tanto de enseñar contenidos teóricos sobre las unidades lingüísticas sino centrar los esfuerzos en que los alumnos desarrollen sus habilidades de comprensión y expresión. Como docentes hay que estar convencido de que el alumnado debe ser un buen usuario de la lengua y no un estudioso teórico de la misma. El conocimiento subconsciente de la lengua (la gramática implícita) es el que ha de fundamentar el desarrollo de la capacidad verbal del alumno y su aprendizaje.

Hay que recordar, aunque sea repetitivo, que la lengua es la herramienta fundamental para la socialización. Actúa como mediadora por excelencia de los procesos de enseñanza-aprendizaje. Facilita, además, la participación de los estudiantes en las rutinas diarias escolares, tanto para interactuar con los compañeros como para acceder a los aprendizajes; permite la interacción con compañeros y adultos en tareas de conversación, descripción, explicación, resolución de problemas interpersonales; facilita la comprensión de los diferentes estilos de discurso y demandas en el aula. El dominio de los aspectos semánticos y morfosintácticos, el aprendizaje de la lectura y la escritura, y el desarrollo cognitivo de estrategias ayudan a la consecución de los diferentes dominios del pensamiento.

Los componentes o niveles del lenguaje fonético-fonológico y léxicosemántico, que representan los pilares fundamentales sobre los que se asienta el proceso de comunicación, parece que son los más susceptibles de análisis, desarrollo o refuerzo. Cuando se adquiere el dominio de la articulación fonética y un nivel de léxico y de vocabulario importantes, fruto de interrelación con los demás, se dispone de mejores condiciones para construir el resto del 'edificio' que es nuestra lengua. 
El desarrollo de la comunicación se verá reforzada y con él la comunicación, el aprendizaje y la maduración personal.

Asimismo, los otros niveles o componentes del lenguaje actúan como complementos necesarios para perfeccionar el dominio de la lengua y para mejorar las prácticas comunicativas. El nivel morfosintáctico de la lengua propicia el dominio de la norma lingüística, el aprendizaje y la automatización de las estructuras que la constituyen, haciendo que los usos comunicativos sean admitidos como correctos y podamos practicarlos en mejores condiciones.

Estos usos comunicativos entran de lleno en el nivel pragmático del lenguaje, nivel en el que cobra relevancia práctica nuestra capacidad de comunicarnos. Si no se sabe o no se es capaz, por ejemplo, de presentarse en público, mantener situaciones de comunicación fluidas, utilizar el lenguaje para obtener información, para resolver situaciones cotidianas en los ámbitos social, familiar o profesional, de nada servirá conocer y dominar un sistema de comunicación, como es la lengua. En este nivel la capacidad creativa asociada al uso de la lengua parece fundamental.

En definitiva, morfosintaxis, semántica y pragmática se constituyen como los niveles claves de una lengua; los dos primeros para dominar sus estructuras y mecanismos; el último para dotar de funcionalidad a ese instrumento de comunicación.

Si se quiere disponer de una herramienta de comunicación útil y práctica, como puede y debe ser la lengua, es fundamental el conocimiento y dominio de los niveles que la constituyen. Nada mejor para ello que aprovechar los procesos de aprendizaje y las etapas educativas obligatorias, cuando existe una mayor plasticidad en las estructuras cerebrales de nuestros alumnos.

\section{Vocabulario, desarrollo del lenguaje y creatividad}

Dentro de la gramática adquiere un valor significativamente muy importante el vocabulario. Es el elemento molecular del lenguaje; es el constituyente esencial en el aprendizaje de las restantes actividades lingüísticas (lectura, escritura, comprensión, expresión, conversación, etc.). La correlación del vocabulario con la inteligencia ya fue mostrada por varios autores (Spearman, 1923; Terman, 1916 o García Hoz, 
1977) y corroborada por estudios mucho más recientes (Murillo, 2009; Piacente et al., 2010; Lojo-Seoane et al., 2014 o Santamaría et al., 2014).

La enseñanza del vocabulario debe ser diferenciada de otros aspectos y niveles del código lingüístico, pero no debe perder su conexión con ellos atendiendo al principio básico de co-implicación lingüística. En este sentido, las palabras (léxico) deben enseñarse con el resto de niveles lingüísticos: (1) el nivel fonético-fonológico que comprende la fonología, o estudio de los fonemas de una lengua, entre los que destaca la fonética, o estudio de la realización alofónica individual de dichos fonemas (los fonos son sonidos del habla, realizaciones diferenciadas de un mismo fonema) y, también, aunque no son campos estrictamente lingüísticos, ya que intervienen factores culturales e históricos, el estudio la grafémica, la ortología y la ortografía.

El segundo nivel que debe relacionarse con el vocabulario o nivel léxico es: (2) el nivel morfosintáctico con la morfología, o estudio de la mínima unidad con significado (el morfema), la palabra y los mecanismos de formación y creación de palabras. Y también la sintaxis, o estudio de la combinatoria sintagmática, en sus dos niveles: el sub-oracional, que corresponde al propio de los Ilamados sintagmas; y el oracional que estudia las relaciones específicas sintagmáticas de los signos lingüísticos que conforman, a su vez, el signo lingüístico gramatical superior del sistema de la lengua.

Resulta muy útil, pedagógicamente hablando, el uso de palabras seleccionadas al azar y su asociación con situaciones u objetos a los cuales se les quiere introducir cambios; el uso de ideas absurdas como fuentes iniciales de pensamiento, las cuales pueden conducir a otras ideas fuera del patrón esperado; la combinación de objetos diferentes para producir algo de mayor significación que la suma de las partes, y el análisis y la búsqueda de discrepancias que generan juicios de valor acerca de las cosas y a la búsqueda de nuevas ideas.

Desde el punto de vista de uso práctico de la lengua, como acción, es importante tomar en consideración no solamente las palabras sino también los textos como unidades superiores de comunicación escrita. Para ello, el alumnado debe sentirse creador, debe experimentar y elaborar su propio material, compartirlo, difundirlo y publicarlo. El alumnado debe experimentar las sensaciones de un escritor o periodista. $Y$ esto puede propiciarse en la clase de lengua y literatura cuando les motivamos para 
redacten algún texto. Como capacidad humana, la creatividad puede ser educada (Martínez Vidal y Díaz Pereira, 2008).

Tomando en consideración todo lo expuesto, se hace una propuesta de actividades lúdicas que sirvan para comprender la norma y código lingüísticos desde una perspectiva lúdica y creativa que fomente la imaginación y curiosidad del alumnado sobre estos niveles o planos lingüísticos al tiempo que adquieren y mejoran su competencia de comunicación lingüística.

Mediante el trabajo individual e intelectual se podrá desarrollar toda la creatividad posible. La iniciativa personal, la curiosidad o el interés por algún aspecto del lenguaje se pueden trabajar en clase siempre que entendamos que el proceso de enseñanza y aprendizaje es, en gran medida, un gran juego y desafío para adquirir conocimientos teóricos y llevarlos a la práctica.

\section{Marco empírico}

\section{Objetivos}

Se propuso desarrollar una experiencia que implicara al alumnado del Grado de Maestro de Educación Primaria de la Universidad de Valladolid, campus de Soria, y escolares de la etapa de Primaria (6-12 años) de seis centros educativos de la ciudad. Los objetivos planteados fueron: despertar la curiosidad y el sentido critico del alumnado participante en la experiencia, tanto universitario como de la etapa de Educacion Primaria; propiciar la reflexión sobre el código linguistico para mejorar la expresión oral y escrita de los escolares; y facilitar la asimilación de diversos contenidos curriculares de forma libre, autónoma y cooperativa. Para ello, la propuesta surgió en la asignatura Didáctica de la creatividad literaria, asignatura optativa de $4^{\circ}$ curso, que se imparte en el primer semestre académico dentro de la mención en Lengua española, literatura y creatividad. Tuvo dos fases diferenciadas: una primera en las aulas universitarias donde se preparó al alumnado universitario y se elaboraron los materiales; y otra posterior en las aulas de los colegios. 


\section{Población y Muestra}

La población/muestra varía según las fases. En la primera participaron dieciocho estudiantes universitarios, para concienciarles sobre la sugestopedia (contexto sugestivo) y la importancia de la creatividad literaria y las actividades lúdicas en los procesos de enseñanza-aprendizaje ya que estos alumnos, futuros maestros, serían después los encargados de aplicarlas en las aulas de Educación Primaria durante el desarrollo de su periodo de prácticas (practicum II). La segunda fase contó con una muestra de 274 escolares que realizaron esas actividades de gramática lúdica y creativa, con el visto bueno y la implicación de doce tutores. Ambos colectivos, estudiantes universitarios y escolares de Educación Primaria, son participantes de la experiencia desarrollada. En la siguiente tabla se recoge la muestra de alumnado de Educación Primaria de los seis colegios:

Tabla 1

Alumnado de primaria participante en la experiencia, según centro, curso y sexo. En gris los cursos seleccionados, al corresponder con los asignados al alumnado de prácticas

\begin{tabular}{|c|c|c|c|c|c|c|c|c|c|c|c|c|}
\hline & \multicolumn{2}{|c|}{ Primero } & \multicolumn{2}{|c|}{ Segundo } & \multicolumn{2}{|c|}{ Tercero } & \multicolumn{2}{|c|}{ Cuarto } & \multicolumn{2}{|c|}{ Quinto } & \multicolumn{2}{|c|}{ Sexto } \\
\hline & $\hat{0}$ & 우 & $0^{\pi}$ & $q$ & $\hat{0}$ & q & $0^{1}$ & 우 & $\hat{0}$ & $q$ & $0^{1}$ & q \\
\hline Colegio 1 & 8 & 13 & 12 & 11 & 9 & 14 & 10 & 14 & 12 & 13 & 11 & 14 \\
\hline Colegio 2 & 6 & 15 & 11 & 10 & 17 & 8 & 9 & 13 & 11 & 12 & 14 & 10 \\
\hline Colegio 3 & 12 & 10 & 11 & 13 & 8 & 17 & 10 & 13 & 8 & 15 & 13 & 11 \\
\hline Colegio 4 & 7 & 14 & 9 & 13 & 10 & 15 & 12 & 12 & 9 & 14 & 6 & 16 \\
\hline Colegio 5 & 9 & 15 & 10 & 12 & 13 & 9 & 9 & 14 & 8 & 16 & 11 & 11 \\
\hline Colegio 6 & 13 & 11 & 13 & 12 & 10 & 15 & 9 & 13 & 13 & 10 & 8 & 14 \\
\hline Totales & 14 & 28 & 23 & 21 & 18 & 32 & 22 & 25 & 21 & 26 & 19 & 25 \\
\hline Globales & \multicolumn{2}{|c|}{42} & \multicolumn{2}{|c|}{44} & \multicolumn{2}{|c|}{50} & \multicolumn{2}{|c|}{47} & \multicolumn{2}{|c|}{47} & \multicolumn{2}{|c|}{44} \\
\hline
\end{tabular}

\section{Instrumento}

Como instrumento se diseñaron desde la asignatura optativa del Grado en Educación Primaria, "Didáctica de la Creatividad Literaria", una serie de actividades de refuerzo a través de una metodología activa, basada en el juego y en la sugestopedia. Siempre fueron actividades consensuadas con los maestros tutores, en momentos que estos consideraron que no distorsionaba el desarrollo normal de las clases, y con una du- 
ración máxima por sesión de 45 minutos, dos días a la semana durante el periodo de prácticas. Al realizarse durante el periodo de prácticas del alumnado universitario, se acordó con el profesor tutor de cada centro de Primaria que decidieran ellos cuándo era el mejor momento para su aplicación en las aulas. La decisión de que se hicieran en periodos donde menos se distorsionara el desarrollo normal de las clases fue sugerida por los propios tutores.

Las actividades diseñadas por los estudiantes universitarios se organizaron en tres bloques (sonidos/letras/sílabas, palabras/frases/oraciones, textos) que, tras un entrenamiento y ajuste entre el alumnado de Grado, fueron aplicadas en las aulas según cada uno de los antiguos tres ciclos de la etapa de Educación Primaria: el primer bloque a $1^{\circ}$ y $2^{\circ}$; el segundo bloque a $3^{\circ}$ y $4^{\circ}$ y el tercer bloque a $5^{\circ}$ y $6^{\circ}$.

Es preciso aclarar que las actividades de refuerzo planteadas formaron parte de la estrategia pedagógica que permitiría mostrar conductas y resultados que se concretarían después en rúbricas de conductas de escolares y tutores, así como en las apreciaciones recogidas en las tablas 2 y 3.

Tabla 2

Rúbrica de evaluación de juegos y actividades de Gramática lúdica

\begin{tabular}{|c|c|c|c|}
\hline \multirow{2}{*}{$\begin{array}{l}\text { Criterios } \\
\text { (puntuación) }\end{array}$} & \multicolumn{3}{|c|}{ Escala de evaluación } \\
\hline & 2 & 1 & 0 \\
\hline Originalidad & $\begin{array}{l}\text { El texto es novedo- } \\
\text { so, humorístico y } \\
\text { creativo. }\end{array}$ & $\begin{array}{l}\text { Alguna parte del tex- } \\
\text { to recuerda a otras } \\
\text { obras. }\end{array}$ & $\begin{array}{l}\text { Todo el texto es } \\
\text { adaptación o copia } \\
\text { de otra obra. }\end{array}$ \\
\hline Esfuerzo & $\begin{array}{l}\text { La extensión es la } \\
\text { solicitada. }\end{array}$ & $\begin{array}{l}\text { La extensión está } \\
\text { muy próxima a la } \\
\text { solicitada, sin llegar } \\
\text { a serlo. }\end{array}$ & $\begin{array}{l}\text { La extensión es } \\
\text { escasa y poco } \\
\text { relevante. }\end{array}$ \\
\hline $\begin{array}{l}\text { Corrección } \\
\text { lingüística }\end{array}$ & $\begin{array}{l}\text { Buena estructura } \\
\text { morfosintáctica, va- } \\
\text { riedad léxica y sin } \\
\text { errores ortográficos. }\end{array}$ & $\begin{array}{l}\text { Limitadas estructuras } \\
\text { morfosintácticas, al- } \\
\text { gún error ortográfico. } \\
\text { Vocabulario común. }\end{array}$ & $\begin{array}{l}\text { Difícil de compren- } \\
\text { der. } \\
\text { Errores ortográficos } \\
\text { frecuentes. Vocabu- } \\
\text { lario inadecuado. }\end{array}$ \\
\hline $\begin{array}{l}\text { Tiempo de } \\
\text { realización }\end{array}$ & $\begin{array}{l}\text { Le sobra tiempo al } \\
\text { realizar la actividad. }\end{array}$ & $\begin{array}{l}\text { Utiliza el tiempo } \\
\text { destinado a la acti- } \\
\text { vidad. }\end{array}$ & $\begin{array}{l}\text { No acaba en el } \\
\text { tiempo establecido. }\end{array}$ \\
\hline $\begin{array}{l}\text { Lectura } \\
\text { en voz alta }\end{array}$ & $\begin{array}{l}\text { Lee de forma ade- } \\
\text { cuada, sin nervios, } \\
\text { alto y claro. }\end{array}$ & $\begin{array}{l}\text { Lectura insegura. } \\
\text { Voz poco clara o sin } \\
\text { volumen adecuado. }\end{array}$ & $\begin{array}{l}\text { No lee en voz alta } \\
\text { ni comparte su } \\
\text { trabajo. }\end{array}$ \\
\hline
\end{tabular}


Sobre la rúbrica, se celebraron varias sesiones previas al periodo de prácticas, donde se informó al estudiante universitario y se unificó el criterio para evitar dispersión de datos o valoraciones diferentes.

Tabla 3

Encuesta de satisfacción para tutores de centros de Educación Primaria

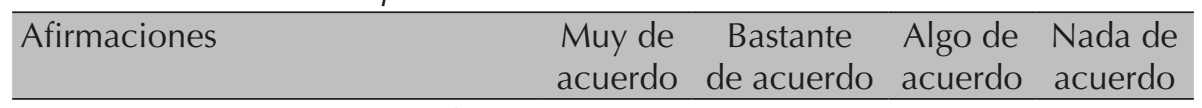

1. Los juegos sirven para hacer más ameno el proceso de enseñanza y aprendizaje.

2. En una sociedad digital es necesario que los alumnos experimenten con letras, palabras, oraciones y textos.

3. Un clima pedagógico afectivo genera más seguridad y entusiasmo entre los alumnos.

4. Es necesario abordar la creatividad en las aulas universitarias y en los Grados de Magisterio.

5. Solo quien ha sido educado en la imaginación es capaz de comprender la realidad.

6. Se deben incluir propuestas de escritura creativa en las aulas de educación primaria.

7. La sugestopedia es una metodología docente importante para desarrollar actividades creativas.

8. La cooperación entre alumnos a la hora de resolver los juegos/actividades es conveniente.

9. La enseñanza de contenidos gramaticales puede ser más asequible si se enfoca de una manera lúdica.

10. El aprendizaje basado en actividades lúdicas permitiría desarrollar destrezas expresivas y comprensivas. 


\section{Procedimiento de recogida y análisis de datos}

Durante el curso 2018-2019, en los seminarios prácticos de la asignatura Didáctica de la creatividad literaria, se plantearon estas actividades como uno de los trabajos prácticos que servirían para evaluar al alumnado del Grado de Educación Primaria; y, por otro, como un estudio aplicado para el que serían protagonistas necesarios. Se explicaron los objetivos que se pretendían conseguir, los materiales que sería necesario diseñar y el proceso de aplicación práctica posterior durante su periodo de prácticas. Igualmente, se acordó mantener reuniones de seguimiento durante su puesta en marcha y de evaluación a la finalización de la experiencia.

Todo el alumnado universitario tomó conciencia de que las letras (sonidos), palabras, frases y textos constituirían la propuesta que se plantearía como tareas de refuerzo para trabajar elementos y estructuras gramaticales propiciando siempre la intervención creativa del alumnado. El trabajo sobre estos niveles o planos lingüísticos se organizó en tres bloques diferenciados con un número equivalente de actividades.

Ya se ha comentado que los pilares básicos sobre los que se asienta el proceso de comunicación se centran en los niveles del lenguaje fonético-fonológico y léxico-semántico y que son los más susceptibles de análisis, desarrollo o refuerzo. La competencia para el dominio de la lengua aumenta cuando se adquiere el dominio de la articulación fonética y un nivel de léxico y de vocabulario importantes, fruto de interrelación con los demás. El desarrollo de la comunicación se verá reforzada y con él la comunicación, el aprendizaje y la maduración personal. El trabajo con las frases, oraciones y texto, con su consiguiente proyección pragmática, también tienen su propuesta.

Los bloques de actividades que se trabajaron en las aulas universitarias quedaron organizados así (ver anexo 1 para conocer su desarrollo en el aula):

a) Juegos con letras para desarrollar la conciencia silábica: familiarización con el golpe silábico; repaso del alfabeto; elaboración de palabras a partir de las letras de otra dada (acróstico); elaboración de palabras con letras interiores de otras dadas (mesóstico); encadenamiento de palabras utilizando la última sílaba; relación de palabras según primera y última sílaba de cada una (bisagra); expresión de palabras con rima y juegos con letras que pueden 
cambiar el significado de las palabras (paronimia).

b) Juegos con palabras: elaboración de textos con palabras que van aumentando progresivamente el número de sonidos (bola de nieve); expresión libre de palabras favoritas, justificando su elección; elección de palabras de un texto para elaborar otro totalmente distinto (explorar y tachar); uso de onomatopeyas en texto de un máximo de cinco palabras; búsqueda de eufemismo de palabras dadas; identificación de palabras dentro de una dada (palabras embarazadas) y elaboración de palabras acortando o alargando una dada (palabras goma).

c) Juegos con textos: interpretación de frases hechas cifradas (refranes); elaboración de textos con palabras que empiezan siempre por la misma letra; creación de texto breves donde estén presentes todas las vocales (pangrama); invención de texto a partir del esquema de la noticia periodística (somos periodistas); creación de historias a partir de parejas de palabras que nada tienen en común (binomio fantástico); elaboración de historias en las que los protagonistas sean los secundarios de cuentos conocidos y debate a partir de expresiones contenidas en cuentos conocidos.

Durante el periodo de prácticas curriculares se mantuvo reuniones con el alumnado participante en la experiencia, para el seguimiento y la introducción de los ajustes necesarios, que siempre fueron mínimos.

Tras el periodo de aplicación de las actividades en las aulas escolares, y a la conclusión del periodo de prácticas del alumnado universitario, se mantuvieron dos reuniones de evaluación en las que se expusieron las observaciones llevadas a cabo, las anotaciones y las valoraciones de lo experimentado.

\section{Resultados}

Se constató en primer lugar una alta implicación de alumnos y tutores de educación primaria, sin la cual no hubiera tenido éxito ni sentido la experiencia desarrollada. Pudo observarse cómo aumentó el grado de participación del alumnado y la motivación a la hora de realizar actividades de aprendizaje. El propio diseño de las actividades, con su elevada dosis lúdica, permitió la participación en las mismas del alumnado 
con retrasos educativos o de quienes presentan necesidades específicas de apoyo, alumnado que habitualmente tiene dificultades para seguir el ritmo normal de las clases.

Además, la realización la mayor parte de las actividades estuvo seguida de puestas en común dentro del grupo clase, que favorecieron el desarrollo de las habilidades lingüísticas orales.

El profesorado tutor de primaria valoró que la realización de estas actividades no supuso interrupción en la dinámica normal de las aulas, pues se escogieron momentos que no estuvieran dedicados a las áreas instrumentales, de manera que estas sesiones siempre fueron percibidas como momentos gratificantes y relajados (lúdicos), aunque estuvieran ayudando a reforzar contenidos gramaticales, contenidos que figuran en el currículum, pero que el alumnado no percibió así.

Por su parte, el alumnado universitario valoró la potencialidad didáctica de las actividades sobre contenidos lingüísticos diseñadas, la manera divertida y creativa con que fueron enfocadas, al tiempo que descubrió una aplicación complementaria a sus prácticas de enseñanza.

Se constató una participación, traducida en interés y realización de las actividades, muy similar entre chicos y chicas y en todos los cursos y centros, sin que se observaran diferencias significativas merecedoras de análisis.

El $82 \%$ del alumnado total de Primaria participante en la experiencia obtuvo la mayor puntuación en el apartado referido al esfuerzo. A partir de tercer curso pudieron valorarse de forma más concreta los demás apartados: es preciso destacar que el 33\% del alumnado alcanzó la máxima puntuación en originalidad, el $68 \%$ en corrección lingüística, el $64 \%$ se adaptó al tiempo requerido para cada actividad y el $72 \%$ en lo que respecta a la lectura en voz alta, compartiendo el trabajo con el resto de la clase.

Respecto al profesorado tutor y al alumnado de prácticas que condujo esta experiencia, los resultados más relevantes fueron los siguientes:

1.- El $79 \%$ del profesorado tutor y el $95 \%$ del alumnado de prácticas manifestó estar muy/bastante de acuerdo en que los juegos son de gran utilidad para hacer más ameno el proceso de enseñanza y aprendizaje. La diferencia que se aprecia es achacable al distinto rol que desempeña un colectivo y otro, pero en todo caso confirma el valor que se concede al empleo de estrategias lúdicas en los procesos de enseñanza y aprendizaje. 
2.- Es igualmente destacable la apreciación respecto a si un clima pedagógico afectivo genera más seguridad y entusiasmo entre los alumnos. El $69 \%$ del profesorado tutor lo estima mucho o bastante, por el $89 \%$ del alumnado universitario en formación. Mientras que este último desarrolla parte de su aprendizaje en este clima, algunos de los maestros tutores que han participado en la experiencia no tuvieron en su momento esa oportunidad, como así lo manifestaron.

3.- Mientras que el $58 \%$ de los maestros tutores se muestra muy/bastante de acuerdo con incluir siempre una propuesta de escritura creativa en las aulas de educación primaria, el porcentaje se eleva al $83 \%$ entre el alumnado en formación, sin duda condicionado por las prácticas que desarrollan en la asignatura Didáctica de la creatividad literaria en la que estaban matriculados.

4.- Respecto a si la sugestopedia es importante como metodología docente en el desarrollo de actividades creativas en el aula, el 61\% del profesorado tutor se mostró muy/bastante de acuerdo, mientras que para el alumnado universitario esta apreciación se elevó hasta el $87 \%$.

5.- Por último, en lo que se refiere a si los procesos de enseñanzaaprendizaje basados en actividades lúdicas o juegos pueden favorecer que el alumnado desarrolle y mejore las destrezas expresivas y comprensivas, el $83 \%$ del profesorado tutor consideró estar muy/ bastante de acuerdo. Entre el alumnado universitario este porcentaje subió hasta el $90 \%$.

\section{Discusiones}

Sobre el valor de los juegos en los procesos de enseñanza y aprendizaje, Guastalegnanne (2009) ya insistió en que despiertan la creatividad y facilitan recursos para que los alumnos se "olviden" de que están trabajando con la lengua y participen en una situación de comunicación real pues "resultan atractivos para todos los estilos de aprendizaje" (p. 2).

En este sentido, los resultados reafirman las tesis de De la Torre (2009) quien ha insistido en la necesidad de abordar la creatividad en las aulas universitarias, sobre todo entre el alumnado que después ejercerá como docente. 
También coincidimos con las tesis de García Carcedo (2011) quien planteó la importancia de incluir siempre una propuesta didáctica de escritura creativa para el desarrollo de la competencia literaria en educación primaria.

Este estudio también reafirma la necesidad de proponer un ambiente en el aula que disponga a la creación literaria del alumnado, tal y como sugiere la sugestopedia (propuesta por Lozanov en 1978). Así como favorecer la autonomía personal, la curiosidad, la experimentación sin miedo a equivocarse, la cooperación entre los compañeros para buscar las mejores soluciones y siempre mediante el diálogo entre el docente y el alumnado. En esta misma línea se enmarcan los estudios realizados por Caro (2006), Guerrero (2008), Freire (2009); Romero (2010); Elisondo, Danolo y Rinaudo (2012); Fernández, Eizagirre, Arandia, Ruiz de Gauna y Ezeiza (2012), Elisondo (2018), Figueroa (2015) y Gómez (2019).

Los resultados confirman el estudio desarrollado por Morote (2014) entre universitarios del Grado de Primaria, tras analizar los resultados de un taller de escritura creativa para profundizar en el proceso de enseñanza-aprendizaje en la formación inicial del Grado de Magisterio. Los procesos de lectoescritura y reflexión sobre la propia lengua ya fueron estudiados por Milla (1999) quien alertó al profesorado de que es un proceso cognitivo complejo que activa estrategias de alto nivel y que requiere una implicación activa y afectiva por parte del sujeto que aprende, confirmando la certeza de que sólo quien ha sido educado en la imaginación es capaz de comprender la realidad.

\section{Conclusiones}

La experiencia desarrollada pone de manifiesto la necesidad de plantear las actividades sobre gramática como si se trataran de juegos creativos pues hacen más ameno el proceso de enseñanza y aprendizaje de los niveles lingüísticos. La experiencia desarrollada confirma que se mejoran el esfuerzo, los rendimientos académicos, el ajuste a unos tiempos determinados, la lectura oral y el trabajo colaborativo.

Es conveniente que los futuros docentes aprendan y fomenten la adquisición de nuevo vocabulario, la expresión fonológica, la sintaxis y el análisis y comprensión de textos. Esto puede facilitarse con una dosis de imaginación y creatividad experimentando con letras, palabras, ora- 
ciones y textos. El alumnado universitario en formación, protagonista de esta experiencia, pudo comprobar y confirmar en unos porcentajes muy elevados que el juego como estrategia, el clima pedagógico afectivo, la propuesta de actividades que impliquen la ejercitación de la escritura creativa y la sugestopedia mejoran los rendimientos académicos, contribuyen a desarrollar las destrezas expresivas y comprensivas del alumnado y facilitan el tratamiento de contenidos gramaticales presentes en el currículum de educación primaria que con frecuencia solo reciben rechazo y frustración, tanto para el alumnado como para el profesorado. Así mismo, el profesorado tutor, cuando apreció la implicación de su alumnado en estas actividades gramaticales creativas y lúdicas, y el rendimiento obtenido de las mismas, lamentó no haberlas incluido en contenidos curriculares ya programados en el área instrumental de lengua castellana.

Por todo lo expuesto, la enseñanza de la gramática puede ser más asequible si se enfoca de una manera lúdica y creativa para que el aprendizaje de los contenidos lingüísticos sea más práctico y natural. Si los docentes optan por esta forma de enseñar, el alumnado desarrollará y mejorará las destrezas expresivas y comprensivas y estará en disposición de elaborar nuevos contenidos, pues habrá reflexionado, además, sobre la norma y el código lingüístico.

\section{Referencias}

Bernal Ruiz, J. A. y Brito Soto, L. F. (2006). 1,2,3 Por todos mis compañeros: algunos juegos de ayer y hoy en México y España. Sevilla: Wanceulen Editorial Deportiva S.L.

Bizquerra Alzina, R. y Pérez Escoda, N. (2007). Las competencias emocionales. Educación XXI, 10, 61-82. https://doi.org/10.5944/educxx1.1.10.297

Caro Valverde, M. T. (2006). Los clásicos redivivos en el aula. Modelo didáctico interdisciplinar en educación literaria. (Tesis doctoral, Universidad de Murcia). Recuperado de http://digitum.um.es/xmlui/handle/10201/117

De la Torre, S. (2006). Comprender y evaluar la creatividad. Málaga: Aljibe.

De la Torre, S. (2009) La Universidad que queremos. Estrategias creativas en el aula universitaria. Revista Digital Universitaria, (10), 12, 1-17. Recuperado de http://www. revista.unam.mx/vol.10/num12/art89/int89.htm

Elisondo, R. M. (2018). Creatividad y educación: Ilegar con una buena idea. Creatividad y Sociedad, (27) 145-166.

Elisondo, R.; Danolo, D. y Rinaudo, M. (2011). Docentes inesperados y creatividad. Experiencias en contextos de educación superior. DOCREA, 1, 103-114. 
Suárez Ramírez, S., Mateos Núñez, M. y Suárez Ramírez, M. (2021). Gramática lúdica y creativa. Una experiencia para hacer más accesibles los contenidos gramaticales en Educación Primaria. Educatio Siglo XXI, 39(3), 187-208.

Fernández Fernández, I., Eizagirre Sagardia, A., Arandia Loroño, M., Ruiz de Gauna Bahillo, P. y Ezeiza Ramos, A. (2012). Creatividad e innovación: claves para intervenir en contextos de aprendizaje. Revista Iberoamericana sobre Calidad, Eficacia y Cambio en Educación, 10, 2, 24-40.

Figueroa, J. (2015). Using gamification to enhance second language learning. Digital Education Review, 27, 32-54.

Freire, Juan (2009). Monográfico "Cultura digital y prácticas creativas en educación". RUSC Universities and Knowledge Society Journal, 6, (1). http://dx.doi.org/10.7238/ rusc.v6i1.23

Fueguel, C. y Montolíu, M. R. (2005). El malestar docente, propuestas creativas para reducir el estrés del profesorado. Barcelona: Octaedro.

García Carcedo, P. (2011). Escritura creativa y competencia literaria. Lenguaje y Textos, 33, 49-59.

García Hoz, V. (1977). Estudios experimentales sobre el Vocabulario. Madrid: C.SIC.

García Velázquez, A. y Llull Peñalba, J. (2009). El juego infantil y su metodología. Madrid: Editex.

Gómez López, A. (2019). The use of escape rooms to teach and learn English at University. En S. Pérez-Aldeguer y D. Akombo (eds.). Research, technology and best practices in Education (pp. 94-102). Eindhoven: Adaya Press.

Guastalegnanne, H. (2009). Juegos para trabajar gramática y vocabulario en la clase ELE. Suplementos marco ELE, 9, 1-34.

Guerrero Ruiz, P. (2008): Metodología de investigación en educación literaria (El modelo ekfrástico). Murcia: DM.

Hidalgo Cabrera, M. (2007). Expresión de la palabra. Lima: San Marcos.

Instituto Cervantes (ed. trad.) (2002). Marco Común Europeo de Referencia de las Lenguas. Recuperado de http://cvc.cervantes.es/ensenanza/biblioteca_ele/marco/cvc_mer.pdf

Labrador Piquer, M. J. y Morote Magán, P. (2008). El juego en la enseñanza de ELE. Glosas Didácticas, 17, 71-84.

Lantigua, J. (2007). El deterioro de los juegos tradicionales. Lecturas: EF y Deportes, 106. Recuperado de http://www.efdeportes.com

Lojo-Seoane, C., Facal, D., Juncos-Rabadán, O. y Pereiro, A. X. (2014). El nivel de vocabulario como indicador de reserva cognitiva en la evaluación del deterioro cognitivo ligero. Anales de psicología, 30, 3, 1115-1121. https://doi.org/10.6018/analesps.30.3.158481

Lozanov, G. (1978). Suggestology and outlines of suggestopedy. Nueva York: Gordon and Breach.

Martí, L. (2010). Los cien lenguajes de la infancia. Cuadernos de Pedagogía, 401, 17-21.

Martínez Vidal, A. y Díaz Pereira, P. (Coord.) (2008). Creatividad y deporte. Consideraciones teóricas e investigaciones breves. Sevilla: Wanceulen Editorial Deportiva.

Milla Lozano, F. (1999): Actividades creativas para la lecto-escritura (Educación Primaria y ESO). Barcelona: Oikos-Tau.

Moreno, N.M; Leiva, J.J; Galván, M.C; López, E; y García, F.J. (2017). Realidad aumentada y realidad virtual para la enseñanza-aprendizaje del inglés desde un enfoque comunicativo e intercultural. En J. Ruiz-Palmero; J. Sánchez-Rodríguez y E.Sánchez- 
Suárez Ramírez, S., Mateos Núñez, M. y Suárez Ramírez, M. (2021). Gramática lúdica y creativa. Una experiencia para hacer más accesibles los contenidos gramaticales en Educación Primaria.

Rivas (Eds). Innovación docente y uso de las TIC en educación. Málaga: UMA editorial. Pp. 1-11.

Morote Peñalver, E. (2014). La escritura creativa en las aulas del grado de primaria. Una investigación-acción. Revista electrónica de Estudios filológicos, 26, 1-25.

Murillo Rojas, M. (2009). Diversidad de vocabulario en los preescolares. Aportes para valorar su competencia léxica. Filología y Lingüística XXXV, 1, 123-138. https://doi. org/10.15517/rfl.v35i1.1271

Nevado Fuentes, C. (2008). El componente lúdico en las clases de ELE. MarcoELE, 7, 1-14. Recuperado de: http://marcoele.com/descargas/7/nevado_juego.pdf

Nussbaum, M. (2012). Sin fines de lucro. Por qué la democracia necesita de las humanidades (Rodil, M. V., trad.). Buenos Aires/Madrid: Katz.

Pérez Escoda, N., Filella Guiu, G., Soldevila, A. y Fondevila, A. (2013). Evaluación de un programa de educación emocional para profesorado de primaria. Educación XX1, 16 (1), 233-254. https://doi.org/10.5944/educxx1.16.1.725

Piacente, T., Fernicola, A. y Piatti, V. (2010). Nivel de vocabulario en alumnos universitarios de psicología. Análisis preliminar. I/ Congreso Internacional de Investigación y Práctica Profesional en Psicología. Facultad de Psicología -Universidad de Buenos Aires, Buenos Aires.

Pisonero del Amo, I. (2004). La enseñanza de español a niños y niñas. En J.Sánchez Lobato, y I.Santos Gargallo, (Dirs): Vademécum para la formación de profesores. Enseñar español como segunda lengua (L2)/lengua extranjera (LE) (pp. 1279-1302).

Rodari, G. (2008). Gramática de la fantasía: introducción al arte de contar historias. (3a ed). Buenos aires: Colihue.

Rodríguez Moreno, M. L. (2006). De la Evaluación a la Formación de Competencias Genéricas: Aproximación a un Modelo. Revista Brasileira de Orientação Profissional, 7 (2), 33-48.

Romero, J. (2010). Creatividad distribuida y otros apoyos para la educación creadora. Pulso, 33, 87-107

Santamaría, P., Arribas, D., Pereña, J. y Seisdedos, N. (2014). EFAl. Evaluación Factorial de las Aptitudes Intelectuales. Madrid: Tea

Sánchez Bolado, J. (2017). El potencial de la realidad aumentada en la enseñanza de español como lengua extranjera. Edmetic, 6 (1), pp.62-80.

Spearman, C. (1923). The nature of "intelligence" and the principles of cognition. London: McMillan.

Terman, L. (1916). The measurement of intelligence. Boston: Houghton Mifflin Company. Vilera, A. (2004). Juegos electrónicos: reto a los procesos educativos y pedagógicos. Acción Pedagógica, 13 (2), 212-227. 
